$\stackrel{-}{=}$

\section{Fostering close encounters of the entomological kind}

Wilson and colleagues (2017) pointed out the lack of basic knowledge of bees (Hymenoptera: Apiformes) among the general public, potentially hindering conservation efforts in spite of massive worldwide support to protect these charismatic organisms. Indeed, insufficient knowledge on biodiversity may undermine conservation actions by overlooking critical requirements of the species at stake (eg setting artificial nest boxes that later become "ecological traps” for owls [Klein et al. 2007], or introducing honey bees that compete with wild bees for limited floral resources [Cane and Tepedino 2017; Geldmann and González-Varo 2018]). Fostering knowledge on pollinator diversity in the general public is challenging given the vast number of species involved: in France alone, more than 950 species of bees, 500 species of hoverflies, 250 species of butterflies, and an unknown but large number of wasp, beetle, moth, and non-syrphid fly species visit flowers and contribute to pollination. In this letter, we argue that ambitious citizen-science monitoring programs can substantially increase biodiversity knowledge in the general public by providing standard protocols to quantify the diversity of flower visitors and ways to share and discuss observations.

As a case study, we analyzed data from the French "Photographic Survey of Flower Visitors", which proposed a standardized protocol not requiring prior entomological knowledge (Deguines et al. 2012). Briefly, survey observers photograph all flower visitors on a freely chosen plant species for at least 20 minutes and then use an identification tool to name each of the photographed insects. The observer has to choose from 593 nonoverlapping insect taxa, which together encompass all arthropod species that can be seen on flowers, including those from the most common taxonomic orders of flower visitors (Diptera, Coleoptera, Hymenoptera, and Lepidoptera). The taxonomic resolution of these taxa includes: "Species from different families" (23 taxa), "A whole family" (34 taxa), "Several genera within a family" (31 taxa), "Species from different genera within a family" (86 taxa), "A genus" (59 taxa), "Species from a genus" (64 taxa), and "A species" (296 taxa). Photographs of the monitored plant as well as each insect taxon and their identifications are shared on a website, with options to comment and update entries. In the survey's first 4 years (2010-2013), 1019 observers provided 65,456 photographic identifications, which were reviewed by experts from a local entomological society (Office pour les insectes et leur environnement [OPIE]; www.insectes.org). We used generalized linear mixed-effects models to test whether image sharing affected the probability of observers correctly identifying the photographed pollinators. We separately analyzed the honey bee (Apis mellifera), the wild bee taxa (42 taxa), the non-bee hymenopteran taxa (51 taxa), the beetle taxa (136 taxa), the butterfly and moth taxa (231 taxa), and the fly taxa (91 taxa), because some groups of flower visitors may be easier to identify than others. Additional methodological details are provided in WebTable 1.

We detected strong positive effects of participation on the probability of correct identification for all flower-visitor groups (Figure 1). The probability of correct identification for honey bees rose markedly, reaching 95\% after 25 photographs; similar (albeit less striking) trends were found

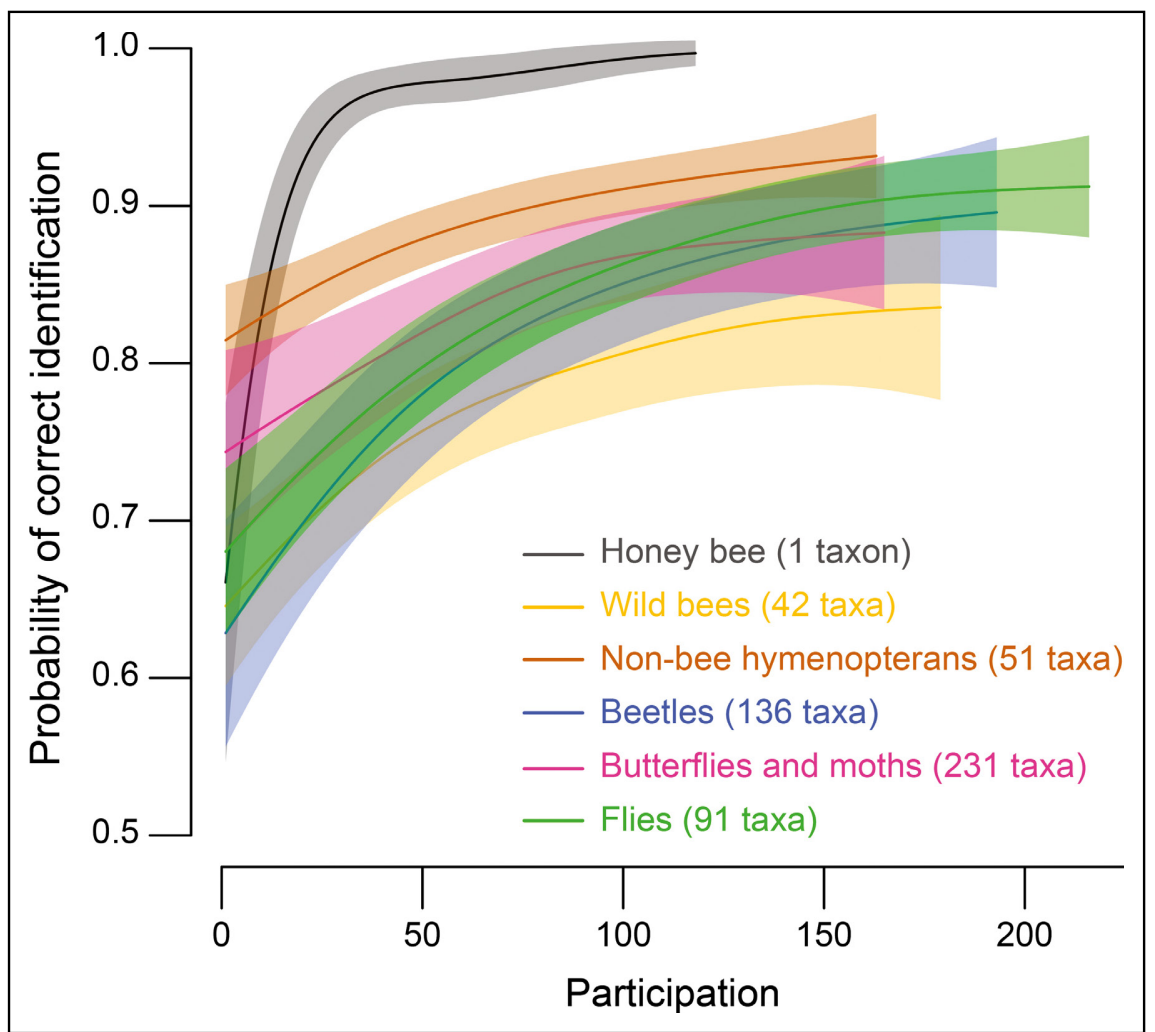

Figure 1. Probability of correct identification improves with participation. Effect of participation (rank of the ith-shared photograph, for a given observer and group of flower visitors) on the probability of correct taxon-level identification of the honey bee (Apis mellifera; gray), wild bees (Hymenoptera: Apiformes, except A mellifera; orange), nonbee hymenopterans (Hymenoptera non-Apiformes; brown), beetles (Coleoptera; blue), butterflies and moths (Lepidoptera; purple), and flies (Diptera; green). Models' sample sizes (number of validated photographs) were 3083, 5339, 3718, 3374, 4028, and 6899 , respectively (WebTable 1). Lines and 95\% confidence intervals (band surrounding each line) were retrieved from generalized additive mixed-effects models using the $R$ package mgcv (Wood et al. 2016) to depict the non-linear relationships more closely. 
among other flower-visitor groups, with a relatively lower rate of correct identification for wild bees (Figure 1). Participation-related benefits on observers' probability of correct identification did not depend on their selfattributed level of entomological knowledge (WebTable 1).

What made our monitoring program so innovative was that it confronted observers with a vast diversity of flower visitors, challenging them to distinguish among 593 taxa (compare with Kremen et al. 2011 and Ratnieks et al. 2016). Despite the difficulty of the task, observers appeared to be motivated by the inherent reward of discovering flower-visitor diversity. By providing observers with an online venue to share their photographs and to discuss each other's contributions, Web 2.0 tools were key to the functioning of the monitoring program and likely contributed to our results by promoting the emergence of an observer community. Between 2010 and 2013, more than 67,000 comments were written by observers having shared at least 30 photographs. We found that observers' level of social integration (measured as the number of submitted and received comments per photograph shared) was positively associated with observers' progress in accurately identifying photographed insects (WebFigure 1). This suggests that belonging to a community of observers helps meet the challenge of better knowing the tremendous diversity of flower visitors, a daunting task for someone facing it on their own. We therefore urge biologists interested in citizen science to not oversimplify their projects, allowing for greater engagement in participation and scientific outcomes. Clear scientific objectives, appropriate tutorials, and regular feedback of results to observers are essential when designing citizenscience projects (Pocock et al. 2015; Cornell Lab of Ornithology 2018).

A range of flying insects - including wild bees - are in critical need of conservation (Goulson et al. 2015; Deguines et al. 2016; Hallmann et al.
2017). Given that participation in monitoring programs appears to favor pro-conservation behaviors (Cosquer et al. 2012), scientifically ambitious citizen-science projects are a powerful tool that biologists and policy makers should embrace in order to achieve positive educational, scientific, and conservation outcomes.

\section{Acknowledgements}

We thank the participants of the Photographic Survey of Flower Visitors, experts (OPIE) who validated the insect identifications, and Headwork (ANR-16CE23-0015) for financial support.

\section{Nicolas Deguines ${ }^{1 *}$, Mathieu de Flores ${ }^{2}$, Grégoire Loïs ${ }^{1}$, Romain Julliard $^{1}$, and Colin Fontaine ${ }^{1}$}

${ }^{1}$ UMR 7204 MNHN-CNRS-UPMC, CESCO, Paris, France

(nicodeguines@gmail.com);

${ }^{2}$ Office pour les insectes et leur

environnement, Guyancourt, France

Cane JH and Tepedino VJ. 2017. Gauging the effect of honey bee pollen collection on native bee communities. Conserv Lett 10: 205-10.

Cornell Lab of Ornithology. 2018. Citizen science toolkit. www.birds.cornell.edu/ citscitoolkit/toolkit. Viewed 15 Jan 2018.

Cosquer A, Raymond R, and PrevotJulliard A-C. 2012. Observations of everyday biodiversity: a new perspective for conservation? Ecol Soc 17: art2.

Deguines N, Julliard R, de Flores M, and Fontaine C. 2012. The whereabouts of flower visitors: contrasting land-use preferences revealed by a country-wide survey based on citizen science. PLoS ONE 7: e45822.

Deguines N, Julliard R, de Flores M, and Fontaine C. 2016. Functional homogenization of flower visitor communities with urbanization. Ecol Evol 6: 1967-76.

Geldmann J and González-Varo JP. 2018. Conserving honey bees does not help wildlife. Science 359: 392-93.

Goulson D, Nicholls E, Botías C, and Rotheray EL. 2015. Bee declines driven by combined stress from parasites, pesticides, and lack of flowers. Science 347: 1255957.

Hallmann CA, Sorg M, Jongejans E, et al. 2017. More than 75 percent decline over 27 years in total flying insect biomass in protected areas. PLoS ONE 12: e0185809.

Klein Á, Nagy T, Csörgó T, and Mátics R. 2007. Exterior nest-boxes may negatively affect barn owl Tyto alba survival: an ecological trap. Bird Conserv Int 17: 273-81.

Kremen C, Ullman KS, and Thorp RW. 2011. Evaluating the quality of citizenscientist data on pollinator communities. Conserv Biol 25: 607-17.

Pocock MJO, Newson SE, Henderson IG, et al. 2015. Developing and enhancing biodiversity monitoring programmes: a collaborative assessment of priorities. J Appl Ecol 52: 686-95.

Ratnieks FLW, Schrell F, Sheppard RC, et al. 2016. Data reliability in citizen science: learning curve and the effects of training method, volunteer background and experience on identification accuracy of insects visiting ivy flowers. Methods Ecol Evol 7: 1226-35.

Wilson JS, Forister ML, and Carril OM. 2017. Interest exceeds understanding in public support of bee conservation. Front Ecol Environ 15: 460-66.

Wood SN, Pya N, and Säfken B. 2016. Smoothing parameter and model selection for general smooth models. J Am Stat Assoc 111: 1548-63.

\section{Supporting Information}

Additional, web-only material may be found in the online version of this article at http://onlinelibrary.wiley. com/doi/10.1002/fee.1795/suppinfo

doi:10.1002/fee.1795

\section{$\triangle$ \\ Urban areas do provide ecosystem services}

Locatelli et al. (2017) characterized how land-use trajectories affect ecosystem services (ES) in mountain landscapes. Despite acknowledging that "urban ecosystems may provide important provisioning services and a variety of cultural services" (Locatelli et al. 2017), their model described urban areas as "Worst-case scenarios (decrease of all services)", with a near-complete absence of provisioning, regulating, and cultural services relative to services in more natural (less developed) areas. Because Locatelli et al. largely focused on the capacity of areas to produce ES, we believe that the authors largely underestimated the flow and demand of ES in urban areas. In response, we wish to clarify that cities are not 